\title{
Why diverse perspectives matter for Memory \& Cognition
}

\section{Ayanna Thomas ${ }^{1}$}

Published online: 27 December 2019

(C) The Psychonomic Society, Inc. 2019

I am happy to announce that this editorial marks a new phase in my relationship with this prestigious journal, as I take the role of editor in chief of Memory \& Cognition (M\&C). My association with $\mathrm{M} \& \mathrm{C}$ began almost 20 years ago when I submitted my first paper for publication. Though I had been warned of the trials and tribulations of the peer-review process, my experience was positive, and the feedback I received from the reviewers at $\mathrm{M} \& \mathrm{C}$ was constructive. I continued to publish in $\mathrm{M} \& \mathrm{C}$ and eventually began serving as an ad hoc reviewer. From reader to author, reviewer, associate editor (AE), and now in the role of Editor-in-Chief, this long and ever-changing relationship has deepened my appreciation and respect for this journal.

Like the editors who came before me, I value the scope and aims of M\&C. The articles published are consistently theoretically important and empirically rigorous. This is owed in large part to the excellent work of the previous editors and editorial teams. The quality of the research published under Neil Mulligan's tenure at M\&C allows me to take over direction of a journal already on the right course - already with a trajectory toward the future of the field. Because of this, stepping up and in as the new editor not only seemed natural but also viable. Therefore, it is with great enthusiasm that I begin my tenure as Editor-in-Chief. I look forward to taking this journey with a team of excellent associate and consulting editors. In this moment, we have the opportunity to improve on an already strong process.

Let me tell you of our plans for the future of M\&C:

\section{Theory development and transparency}

$\mathrm{M} \& \mathrm{C}$ already has a reputation for publishing strong, theoretically grounded empirical research. As editor, I plan to carry

Ayanna Thomas

ayanna.thomas@tufts.edu

1 Psychology, Tufts University, Medford, MA, USA on this important tradition, while recognizing the difficulty of developing rigorous theories. A single manuscript is unlikely to build a theory that will affect how subsequent studies are developed and how hypotheses are tested (Gray, 2017). An individual manuscript, whether presenting what may be considered exploratory or confirmatory research, may provide a piece of the puzzle necessary for understanding the picture hidden within. $M \& C$ will continue its practice of publishing empirically focused research with the aim of developing the components necessary for precise theory development. The development of pet theories generated from siloed research agendas have long plagued the field, and we are at an exciting time where we have the tools and will to address this scientific constraint. The new editorial team at M\&C sees as one of its primary missions to emphasize and promote science that establishes specific and clear links between underlying constructs. To achieve this mission, we encourage the submission of research that independently adds to emerging theories and also presents an architecture for those emerging theories. This mission will be achieved by including both confirmatory and exploratory research. Both are important for developing strong theory. That said, neither is valuable unless they are based upon robust and reliable methods.

How do we know which methods are reliable? The answer has always governed the mission of $\mathrm{M} \& \mathrm{C}$, which is to publish reproducible research that reports experiments buttressed by robust designs and methods. Understanding the reliability of methods also means that null results should be considered. Therefore, I will continue the practice of publishing experiments with strong research designs that treat not only participants but also the stimuli as random variables. Statistical significance will continue to be evaluated, but will not be a necessary requirement for publication. Rather, I firmly believe that multiple experiments are required to develop reliable methods and establish a predictable pattern of results. These values are consistent with the underlying principles driving the preregistration movement (e.g., Nosek, Ebersole, DeHaven, \& Mellor, 2018), but like many others (e.g., Oberauer \& Lewandowsky, 2019; Szollosi et al., 2019), I am confident that preregistration alone will not yield reliable research and strong theory. This is why a strong editorial team 
with a commitment to these ideals will allow $\mathrm{M} \& \mathrm{C}$ to act within its mission moving forward.

Whether confirmatory or exploratory, science can only benefit if we all adopt practices whereby our materials, procedure, and raw data are available openly. More than just by request, as incoming editor, I would like to encourage authors to consider the value of these transparent practices. Though we will neither require data to be open nor a study to be preregistered to be considered for publication, I invite future authors to consider whether either or both would improve not only the evaluation of research but the questions that others pose once that research is disseminated.

\section{Fresh perspectives}

Research in topics covered in $\mathrm{M} \& \mathrm{C}$ has made great strides in moving the field in interesting and innovative directions. However, old problems persist. For example, we remain stagnant in our ability to separate the contributions of encoding and retrieval on long-term retention. We continue to struggle with how we understand constructs such as attention and working memory. We often retread over areas that have been well studied with the methods and approaches available. As a field, we must embrace examining problems from multiple angles and accommodate conflicting perspectives. As we have recently seen, design (Landy et al., 2019) and analysis (Silberzahn et al., 2018) choices have a dramatic impact on results. These findings not only highlight the need to be open and detailed about our design choices but also point to the need for more studies that evaluate the same research question using different methods (Fiedler, 2017). In terms of building solid theory, $\mathrm{M} \& \mathrm{C}$ will consider experiments that vary stimuli and study design, and replicate findings across more geographic locations and populations.

\section{Diversity of thought}

Meta-theory development will, in part, be the result of diversifying our approaches to the study of memory and cognition. As editor, I would like to increase the diversity of research questions (e.g., interdisciplinary, and inter-subdisciplinary questions) and approaches (e.g., computational, individual differences) published in M\&C. Topics and approaches within the content areas of memory and cognition are numerous, yet my experience as an $\mathrm{AE}$ suggests that articles submitted and published cover only a small fraction of these topics. There are several reasons why I think this may be the case, but rather than explore these possibilities, I intend to present mechanisms that will allow us to increase the scope of research published in M\&C.
Editorial boards are often demographically, culturally, and/ or geographically homogenous. This homogeneity results in convergence on a narrow suite of research topics and approaches that the team considers worthy of publication. The board structure may unintentionally promote a narrow perspective. Indeed, this is among the principal reasons put forward to explain why women and people from historically marginalized populations remain severely underrepresented on editorial boards across academic fields.

Having editorial boards that reflect this increasing intellectual, demographic, geographic, and cultural diversity of the global scientific community will benefit the journal and the field more broadly. Scientists trained in different parts of the world can also have very different epistemological orientations. Increasing the diversity on an editorial board could therefore, broaden the scope of theoretical and methodological approaches that M\&C publishes. Ultimately, increasing editorial diversity should help to minimize biases in the review, publication, and citation of articles based on an author's nationality, home country, gender, and/or cultural identity.

The development of the incoming team was based in part on these important observations. The 10 member AE team consists of scholars who offer diversity in perspectives with expertise in event cognition, visual attention, working memory, and collaborative remembering, to name only a few. I encourage the reader to take a close look at this team, as they are the backbone of this journal. Similarly, the 48 members of the consulting editors (CE) team increase the intellectual and geographical diversity of those who are working to uphold the high standards of M\&C. This team represents researchers from 10 countries, and, notably, nearly $45 \%$ of this team's members resides outside of the United States.

\section{Enabling the team}

The goal of an editor of any scientific journal should be to publish high-quality research that will have an impact on the field. This is not a novel idea. However, training AEs and reviewers to identify high-quality research, and guiding authors in revising manuscripts to highlight contributions without going beyond what the data suggest, is more challenging. AEs and reviewers receive little to no direct training or feedback on their performance. This limitation can be corrected by an editor who provides regular feedback. Such feedback will have direct consequences on the peer-review process by improving the quality of published research and by directly improving the skills necessary for reviewing and deciding on value to our readership. I firmly believe continued training and feedback to AEs, CEs, and reviewers are crucial to improving the value of the peer-review process. Additionally, recognition of this work is important. As editor, I will spearhead efforts to recognize associate and consulting editors for 
their service. I will also initiate similar efforts toward the end of recognizing reviewers for their service.

The goal for AEs is to identify and train reviewers to effectively criticize with the aim of improving the manuscript, and avoiding unnecessary negativity. As editor, I will develop and disseminate guidelines for reviewers and AEs. These guidelines will include information about how to effectively critique and how to determine whether a manuscript can be improved to meet the criteria for publication. The editor has the responsibility of setting the tone, establishing the criteria for evaluation, and providing a framework for constructive and effective criticism. This happens through training and discussion, both of which require time and effort. However, the result will be one in which the peer-review process is strengthened. Although every editor has a unique vision, the guidelines and rubrics generated under my tenure will remain available for future use.

\section{What authors and readers should expect}

Authors should expect high-quality, fair reviews delivered in a timely fashion. Even in the unlikely case of a "desk-rejection," where I may decide not to send a manuscript out for full peer review, I will provide feedback. Readers and authors should expect that $\mathrm{M} \& \mathrm{C}$ will continue to produce high-quality content. However, they should also count on an increase in diversity in approaches and perspectives. Importantly, I do not see this as a fundamental change in what is stated as the scope and aims of the journal. Rather, I see that diversifying the team will result in increasing the kinds of submissions $\mathrm{M} \& \mathrm{C}$ receives.

I am confident that diversity in perspectives, training, feedback, and transparent processes in our scientific endeavors will foster improvements in psychological science. The pervasive theme through this opening editorial is one in which we, as a community, work together for the advancement of our understanding of memory and cognition. I am so excited that we are on this journey together.

\section{References}

Fiedler, K. (2017). What constitutes strong psychological science? The (neglected) role of diagnosticity and a priori theorizing. Perspectives on Psychological Science, 12(1) 46-61. https://doi.org/10.1177/ 1745691616654458

Gray, K. (2017). How to map theory: Reliable methods are fruitless without rigorous theory. Perspectives on Psychological Science, 12(5) 731-741. https://doi.org/10.1177/1745691617691949

Landy, J. F., Jia, M., Ding, I. L., Viganola, D., Tierney, W., Dreber, A., ... Uhlmann, E. L. (2019). Crowdsourcing hypothesis tests: Making transparent how design choices shape research results. Psychological Bulletin.

Nosek, B. A., Ebersole, C. R., DeHaven, A. C., \& Mellor, D. T. (2018). Proceedings of the National Academy of Sciences of the United States of America, 115(11) 2600-2606. https://doi.org/10.1073/ pnas. 1708274114

Oberauer, K., \& Lewandowsky, S. (2019). Addressing the theory crisis in psychology. Psychonomic Bulletin \& Review, 26(5), 1596-1618. https://doi.org/10.3758/s13423-019-01645-2

Silberzahn, R., Uhlmann, E. L., Martin, D., Anselmi, P., Aust, F., Awtrey, E., ... Nosek, B. A. (2018). Many analysts, one data set: Making transparent how variations in analytical choices affect results. Advances in Methods and Practices in Psychological Science, 1, 337-356. https://doi.org/10.1177/2515245917747646

Szollosi, A., Kellen, D., Navarro, D. Shiffrin, R. Rooij, I. Zandt, T., \& Donkin, C. (2019). Preregistration is redundant, at best. https://doi. org/10.31234/osf.io/x36pz.

Publisher's note Springer Nature remains neutral with regard to jurisdictional claims in published maps and institutional affiliations. 